\title{
Peritoneal Dialysis Utilization Globally and Opportunities in Lower Income Countries
}

\author{
Fredric O. Finkelstein ${ }^{1}$ \\ Qamar Khan² \\ ${ }^{1}$ Clinical Professor of Medicine, Yale University, New Haven, CT USA \\ ${ }^{2}$ Evansville Veterans Administration Healthcare Center, Evansville, IN USA
}

\section{Correspondence:}

Fredric Finkelstein

136 Sherman Avenue

New Haven, CT 06511, U.S.A.

Email:fof@comcast.net

\author{
PJKD 2020;4(supplement 1):3-5
}

There has been an expansion of peritoneal dialysis (PD) utilization globally over the past several years. This has occurred for several reasons. First, there has been a global increase in the number of patients receiving end-stage kidney disease (ESKD) treatment in high income, middle income and low income countries. ${ }^{1}$ Second, recent studies have emphasized the reduced cost of PD compared to hemodialysis (HD) if PD supplies can be acquired at a reasonable cost. ${ }^{1,2}$ Thirdly, it is now widely accepted that since PD is much simpler to do than $\mathrm{HD}$ (that is, it does not require large amounts of water, complex water treatment systems, electricity, and machinery), the use of PD in low resource countries has certain obvious advantages. ${ }^{2}$ Fourthly, it has become clear from experiences in Hong Kong and Thailand that have developed PD First programs (i.e. the government paying for ESKD care only if PD eligible patients start on PD rather than HD) and programs in Mexico (where there has been limited availability of HD centers) that the vast majority of patients with ESKD, even in low resource countries, are able to successfully be cared for with PD. ${ }^{1-4}$ And, importantly, as programs expand in low resource countries and experience is gained, outcomes of PD improve. ${ }^{3}$ Lastly, the International Society of Peritoneal Dialysis (ISPD) has developed comprehensive guidelines for the care of PD patients that has resulted in a dramatic improvement in outcomes for PD patients over the last several years. ${ }^{1,5}$

The percentage of ESKD patients maintained on PD varies widely globally as illustrated in Figure 1. ${ }^{1}$ In Hong Kong, which has had a PD First program for several years, about $75 \%$ of ESKD patients are maintained on PD (1) In Thailand, which started a PD First program more recently, the percent of patients maintained on PD has been steadily increasing and is currently running about $50 \%$. ${ }^{1}$ On the other hand, in Japan, the percentage is extremely low $(<5 \%)$; the reasons for this are not clear but may reflect government reimbursement policies of dialysis care. In the United States, there has recently been an increase in the percentage of patients maintained on PD in response to changes in reimbursement policies and encouragement by the government to expand home ESKD therapies. ${ }^{1}$ But in countries with well organized education programs for patients with chronic kidney disease (CKD) (such as the United Kingdom, Australia, Canada, the Netherlands, and Belgium), about 25\% of ESKD patients are maintained on PD. ${ }^{1}$ This reflects patient choice when differing modalities are presented to patients and their families -- presumably in an unbiased fashion - and patient decisions are made with shared-decision making.

As countries decide to expand coverage for patients with ESKD, various factors need to be taken into account regarding expansion of HD and/or PD facilities, including the impact on patients, complications of therapy, logistical and technical issues, and financial issues.

What is now well established is that there do not appear to major differences in mortality rates or standard health related quality of life measures (HRQOL) between patients maintained on HD and PD ; but patients are more satisfied with PD than HD and PD has less of a negative impact on patients' lives.

For patients, there are challenges of each treatment and complications of the two therapies are different. PD patients need to do the treatment themselves (or with family or others assisting) and adhere to detailed instructions about how to perform the dialysis. They need to have clean areas to do exchanges and an appropriate place to store the PD supplies. Arrangements need to be made to deliver supplies to patients' homes, which can potentially be problematic for patients living in remote areas. Peritonitis remains a major complication, although peritonitis rates have decreased dramatically over the last several years and are now averaging about 1 infection every 24-72 months of treatment. ${ }^{7}$ Although peritonitis can often be treated at home, appropriate support services are needed since it is important to obtain cultures to define the offending organism and some patients require hospitalization; there is clearly a mortality (albeit low) associated with peritonitis. ${ }^{8}$ Technique failure with PD occurs in about 10-15\% of patients annually requiring transfer to HD. ${ }^{9}$ The most common reasons for transfer include peritonitis, loss of ultrafiltration, inadequate clearances, and various psychosocial reasons. Thus, it is essential that adequate HD support be available when developing and expanding PD programs to accommodate those patients requiring transfer to center HD. Placement of a PD catheter is generally not difficult and can be done by nephrologists as well as surgeons and interventional radiologists. ${ }^{10}$ 


\section{PD Utilization: Cost Analysis}

Figure 1: Percent distribution of prevalent dialysis patients, by modality, from the USRDS 2018

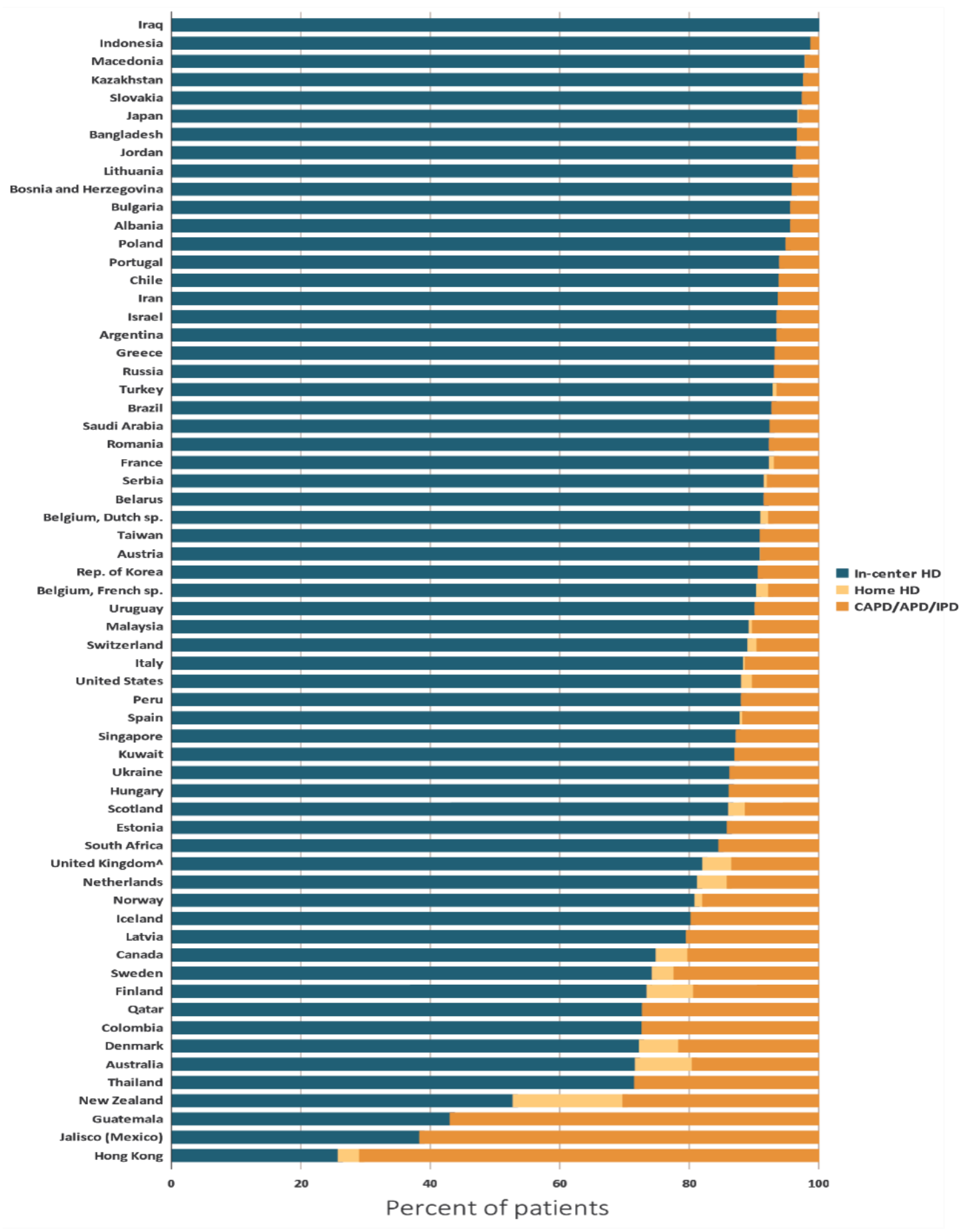

Problems with HD treatments include transportation to and from the dialysis facility and adherence to a rigid dialysis schedule which can be expensive, interfere with work, and/or interfere with family activities. Two times/week dialysis (which is often the standard of care in low income countries) may not provide adequate dialysis (in terms of solute removal and ultrafiltration), particularly in patients who have lost residual renal function. ${ }^{7}$ This can significantly compromise patient HRQOL (7) Post dialysis fatigue can be a major problem. ${ }^{7,11}$ Patients on average require 6 hours to be able to resume normal activities after each HD session. ${ }^{11}$ And, HD requires a vascular access, which can be challenging and requires adequate surgical and radiological support, which can present problems in low-middle income countries (LMIC). 


\section{PD Utilization: Cost Analysis}

PD has a number of features that are attractive in LMICs. These include few technical demands, use in remote regions, reduced need for trained staff and low cost to establish programs. Practical problems in expanding PD programs include training individuals to train and support patients and place catheters and the ability to obtain dialysate and catheters at an acceptable cost. The local manufacture of PD solutions in LMIC has not proved to be practical because it may be difficult to achieve an adequate volume of business to make the program financially solvent and manufacturing solutions with appropriate connection devices, which are essential to reduce peritonitis rates, has proven to be challenging. ${ }^{7}$ It is essential that a reliable source of PD solution be available for all patients.

The success of PD programs depends very much on adhering to international standards of care. The ISPD has played a key role in setting these basic standards, which are freely available on its website. ${ }^{2}$ Adherence to these standards has resulted in sustained improvements in outcomes for PD patients over the past several years.

Economic considerations are important, and studies generally suggest that the cost of PD treatment is less than HD. . $^{1,7}$ Actually, determining costs of providing ESKD care has proven to be challenging. From a facility point of view, costs need to take into account the costs of constructing facilities, acquiring machines, paying for water and electricity, depreciating and repairing equipment, and paying staff. From a patient point of view, costs include the price of medications, transportation to and from dialysis facilities, the cost of lost employment, the cost of family members providing support, etc. From a health system point of view, the cost of care should include not just the costs of dialysis but the total cost of care, including hospitalizations, all medications, access procedures, co-morbid illnesses, etc. In the United States, dialysis accounts for about $40 \%$ of the total costs or providing care for ESKD patients. ${ }^{1}$ A recent publication from Hong Kong is of particular interest. This study compared the costs of peritoneal dialysis and center hemodialysis for the first two years of therapy for patients in Hong Kong, focusing on societal/patient and health service providers' costs. The societal/patient costs included not only the direct health costs but also the transportation costs, loss of patient productivity, caregivers' time and costs, etc. Using this formulation, societal/patient costs for HD were HK\$ 452,151 and 413,017 for the first and second years respectively compared to HK\$ 189,191 and 151,520 for PD. Clearly, PD was much less expensive. How this translates into costs in LMIC countries needs further study. But this study emphasizes the importance of taking into account not just the direct costs of dialysis, but the cost on the patient, his/her family, and society in assessing the financial burden of ESKD care.

In summary, PD offers a cost-effective and viable option to expand ESKD services in LMIC countries. Experiences around the world and from international organizations can provide guidance concerning the establishment of PD programs from a medical and economic standpoint.

\section{References:}

1. United States Renal Data system, 2019

2. Harris DH, Davies SJ, Finkelstein FO et al. Increasing access to integrated ESKD care as part of Universal Health Coverage. Kidney Int. 2019 95(4S):S1- S33,

3. Wong CKH, Chen J, Fung SKS, Mok MMY, Cheng YL, Kong I, Lo WK, Lui SL, Chan TM, Lam CLK Direct and Indirect costs of end-stage renal disease patients in the first and second years after initiation of nocturnal home hemodialysis, hospital hemodialysis, and peritoneal dialysis. Nephol Dial Transplan 34: 1565-1526, 2019

4. Changsirikulchai S, Sriprach S, Thokanit NS, Janma J, Chuengsaman P, Sirivongs D. Survival Analysis and Associated Factors in Thai Patients on Peritoneal Dialysis Under the PD-First Policy. Perit Dial Int. 2018 May-Jun;38(3):172-178

5. www.ISPD. org; education and guidelines

6. Juergensen Wuerth D, Finkelstein SH,. Juergensen PJ, Bekui A, and Finkelstein FO. Hemodialysis and Peritoneal Dialysis: Patients' Assessment of Their Satisfaction with Therapy and the Impact of the Therapy on Their Lives. Clin J Am Soc Nephrol 1: 1191-1196, 2006

7. Sola L, Levin NW, Johnson DW et al Development of a Framework for Minimum and Optimal Safety and Quality Standards for Hemodialysis and Peritoneal Dialysis. Kidney Int Reports, 2020 in press

8. Fried LF, Bernardini J, Johnston JR, Piraino B. Peritonitis influences mortality in peritoneal dialysis patients. J Am Soc Nephrol. 1996 Oct; 7(10):2176-82.

9. Afolalu B, Troidle L, Osayimwen O, Bhargava J, Kitsen J Finkelstein FO: Technique Failure and Center Size Technique Failure and Center Size in a Large Cohort of PD Patients in a Defined Geographic Area. Perit Dial Int. 2009;29:292-6

10. Gorbatkin C, Bass J, Finkelstein FO, Gorbatkin S: Peritoneal Dialysis in Austere Environments: An Emergent Approach to Renal Failure Management: West J of Emerg Med. 2018;19(3):548-556

11. Lindsay RM, Heidenheim PA, Nesrallah G, Garg AX, Suri R; Daily Hemodialysis Study Group London Health Sciences Centre. Minutes to recovery after a hemodialysis session: a simple health-related quality of life question that is reliable, valid, and sensitive to change. Clin J Am Soc Nephrol. 2006 1:952-9 